\title{
A multi-biomarker disease activity score for monitoring rheumatoid arthritis
}

\author{
This article was published in the following Dove Press journal: \\ Current Biomarker Findings \\ 6 October 2015 \\ Number of times this article has been viewed
}

\section{Shintaro Hirata' \\ Nadine Defranoux ${ }^{2}$ \\ Kentaro Hanami' \\ Kunihiro Yamaoka ${ }^{3}$ \\ Yoshiya Tanaka'}

'The First Department of Internal Medicine, School of Medicine, University of Occupational and Environmental Health, Kitakyushu, Japan; ${ }^{2}$ Crescendo Bioscience, South San Francisco, CA, USA; ${ }^{3}$ Division of Rheumatology, Department of Internal Medicine, Keio University School of Medicine, Tokyo, Japan
Correspondence: Yoshiya Tanaka The First Department of Internal Medicine, School of Medicine, University of Occupational and Environmental Health, I-I Iseigaoka, Yahatanishi, Kitakyushu, 807-8555, Japan

Tel +8I 93603 I6II

Fax +8I 936919334

Email tanaka@med.uoeh-u.ac.jp
Abstract: Rheumatoid arthritis (RA) is a chronic, systemic inflammatory disease that affects the synovium and results in cartilage degradation, bone erosions, and joint deformities. RA-associated pain, decreased mobility, fatigue, and comorbidities lead to functional disability, impaired quality of life, and shortened life expectancy by 5-10 years. According to the US Centers for Disease Control, RA ranked as the 42nd highest contributor to global disability, with a prevalence of 1.5 million in the USA and 1.24 million in Japan. Synthetic and biologic disease-modifying anti-rheumatic drugs have improved patient health and disease outcomes. Early diagnosis and intervention to control and decrease disease activity have led to improved patient outcomes. Furthermore, the emergence of a treat-to-target strategy and the definition of remission criteria by the American College of Rheumatology and European League Against Rheumatology have provided a new framework for physicians to achieve better patient outcomes based on regular evaluation of disease activity and assessment of the response to treatment. Improvement is needed, however, in facilitating disease activity assessment and identifying patients at higher risk of radiographic progression and those with smoldering disease who could benefit from more aggressive intervention. An objective disease activity test based on biomarkers measured in the blood that reflects the underlying biological events in addition to information on risk of radiographic progression would fulfill this need. Such a test would provide physicians with a convenient measurement tool to monitor patients in a clinical setting and support rapid treatment adjustment and tighter disease control. This article examines peer-reviewed publications cited in PubMed that describe the multi-biomarker disease activity score, its development and validation, and its applications as an objective disease assessment tool in patients with RA.

Keywords: algorithm, multi-biomarker disease activity score, disease activity, rheumatoid arthritis, Vectra DA

\section{Background}

Rheumatoid arthritis (RA), a systemic inflammatory disease, is a chronic debilitating condition that affects the synovium of the joints, resulting in cartilage degradation and bone erosions, leading to deformities of the joints. The pain, decreased mobility, fatigue, and comorbidities associated with RA lead to functional disability, impaired quality of life, and shortened life expectancy by 5-10 years.

In a 2014 article, Cross et al ranked RA as the 42nd highest contributor to global disability, just below malaria. ${ }^{1}$ In Japan, the prevalence of RA was estimated to be 1.24 million in $2010 .^{2}$ In the USA, according to Centers for Disease Control statistics, the 2005 prevalence of RA was estimated to be approximately 1.5 million $(0.5 \%-1.0 \%$ of the population). ${ }^{3}$ 
Synthetic and biologic disease-modifying anti-rheumatic drugs (DMARDs) such as methotrexate (MTX) and inhibitors of tumor necrosis factor (TNF)-alpha, have contributed over the past decades to dramatically improving patient outcomes, although treatment efficacy can vary widely across individuals and unresponsiveness can develop over time. Early diagnosis and intervention targeted toward controlling disease activity and reaching and maintaining clinical remission or low disease activity have proven to be efficient strategies to improve outcomes. ${ }^{4,5}$ In this context, repeated disease activity assessments have emerged as an important tool. Monthly assessment of disease activity for patients with moderate to high disease activity, and every 3-6 months for patients with sustained low disease activity or remission, is currently recommended. ${ }^{6}$

The importance of disease activity monitoring was also confirmed in several landmark studies assessing the benefit of treat-to-target strategies, including Tight Control of Rheumatoid Arthritis (TiCoRa), Computer Assisted Management for Early Rheumatoid Arthritis (CAMERA), and BeSt (Dutch acronym for Behandel-Strategieen, "treatment strategies"). ${ }^{7-9}$

Disease activity measures such as the 28-joint disease activity score (DAS28), clinical disease activity index (CDAI), simple disease activity index (SDAI), markers of joint damage such as radiographs, physical function assessment by the Health Assessment Questionnaire Disability Index (HAQ-DI) and remission criteria as defined by the American College of Rheumatology (ACR) and European League Against Rheumatology (EULAR), have provided a framework for physicians to evaluate disease activity in patients and their response to treatment. ${ }^{10-13}$ These disease activity measures are based, at least in part, on subjective measures such as patient and physician global assessments, and joint counts. However, these scores have had limited value in assessing disease prognosis, and a need remains to better identify patients at higher risk of radiographic progression or with smoldering disease who would benefit most from early and aggressive intervention, and early assessment of treatment efficacy.

A disease activity score based on serum biomarkers provides the benefit of being objective while simultaneously capturing the pathological processes at a molecular level. In addition, such a biomarker-based disease activity score allows frequent patient monitoring in a clinical setting, and thus supports rapid treatment adjustment and tighter disease control.
Erythrocyte sedimentation rate (ESR) and C-reactive protein (CRP) have shown correlations with disease activity and have proven useful for assessing the clinical response to treatment. ${ }^{14}$ Although these are widely used to determine disease activity in patients with RA, alone or as part of the DAS calculation, they present the shortcomings of being systemic markers of inflammation, and thus, non-specific for RA, and of being affected by many external factors. In addition, ESR is not a very sensitive parameter, and CRP, although more sensitive, is often found within normal ranges in patients with active disease who are treated with DMARDs or biologics. ${ }^{15,16}$ Moreover, neither ESR nor CRP can predict the subsequent risk of disease progression in these patients.

Numerous individual biomarkers have been shown to be associated with disease activity; however, they often lack robustness and fall short of representing the complexity of the disease.$^{17}$ In a 2014 review on biomarkers for early diagnosis of RA, Chandrashekara speculates that, in a complex disease like RA, it is improbable that a single unique biomarker will be identified to predict development of the disease. ${ }^{18} \mathrm{In}$ line with that perspective, a multi-biomarker disease activity (MBDA) score, determined from the concentration of 12 serum biomarkers, was developed by Crescendo Bioscience to measure RA disease activity. ${ }^{19-21}$

This review article examines peer-reviewed publications cited in PubMed that describe the development and validation of the MBDA test, and its applications as an objective disease assessment tool in patients with RA. These publications can be categorized into two groups of studies: those that describe the development and validation of the MBDA score and its correlation with other disease activity measures; and those that address use of the MBDA score in patients with early or established RA, treated either as part of a clinical trial or standard of care.

\section{MBDA score development and validation}

The MBDA score, which ranges from 1 to 100 , was developed to offer clinicians a measurement tool to easily and objectively assess disease activity in patients diagnosed with RA that would correlate with clinical measures commonly used in clinical trials. An additional intent was to capture the complex biological systems affected by the disease. This quantitative score is calculated using an algorithm that combines the concentration of 12 serum biomarkers. The 12 biomarkers and statistical algorithm were determined by studying $>1,700$ patients ( $>1,900$ samples) from multiple cohorts. The algorithm was 
trained through a multi-step process using DAS28-CRP as the reference (Figure 1). 19,20,22 The strength of this approach is the large and independent cohorts of patients used and the statistical modeling methodologies applied.

The 12 biomarkers (vascular cell adhesion molecule 1 [VCAM-1], epidermal growth factor [EGF], vascular endothelial growth factor A [VEGF-A], interleukin 6 [IL-6], TNF-alpha receptor $1 \mathrm{~A}$ [TNF-R1], leptin, resistin, matrix metalloproteinases [MMP] 1 and 3, human cartilage glycoprotein 39 [YKL-40], serum amyloid A [SAA], and CRP, Table S1) are measured by immunoassays using multiplex panels on the Meso Scale Discovery platform. The following MBDA categories were validated: low disease activity, MBDA score <30; moderate disease activity, MBDA score 30-44; and high disease activity, MBDA score $>44$. These cut-offs were determined based on the corresponding DAS28-CRP values ${ }^{23}$ using the relationship specified in the MBDA algorithm: MBDA = round $(\mathrm{DAS} 28-\mathrm{CRP} * 10.53+1)$, and validated independently. ${ }^{19,20}$ Table 1 illustrates the clinical characteristics of patients based on this MBDA score classification in validation studies. Based on the same mathematical reasoning, MBDA scores $<26$ would be associated with disease in remission, although this threshold has yet to be formally validated. ${ }^{19,24,25}$

\section{Correlation of MBDA score with other measures of disease activity}

The MBDA score was shown to correlate with current clinical disease activity measures in several cross-sectional analyses. The first statistically significant correlations of the MBDA score with DAS28-CRP, SDAI, CDAI, and Routine Assessment of Patient Index Data 3 were reported by Curtis et al, who studied a validation cohort of 426 patients; significant associations were observed in both seropositive and seronegative patients (Table 2). ${ }^{20}$ These results were corroborated by the analyses reported in early RA patients from the BeSt cohort. ${ }^{24}$ In this work, a cross-analysis of 179 patient visits showed significant correlations between the MBDA score and DAS28-ESR ( $r=0.66)$, SDAI $(r=0.67)$, CDAI $(r=0.56)$, and HAQ-DI ( $r=0.50$ ), all with $P<0.0001$ (Figure 2 ). A correlation between MBDA score and DAS28-CRP was also reported in the early RA patient population from the Computer Assisted Management in Early Rheumatoid Arthritis cohort. $^{21}$ In this study, performance of the MBDA score

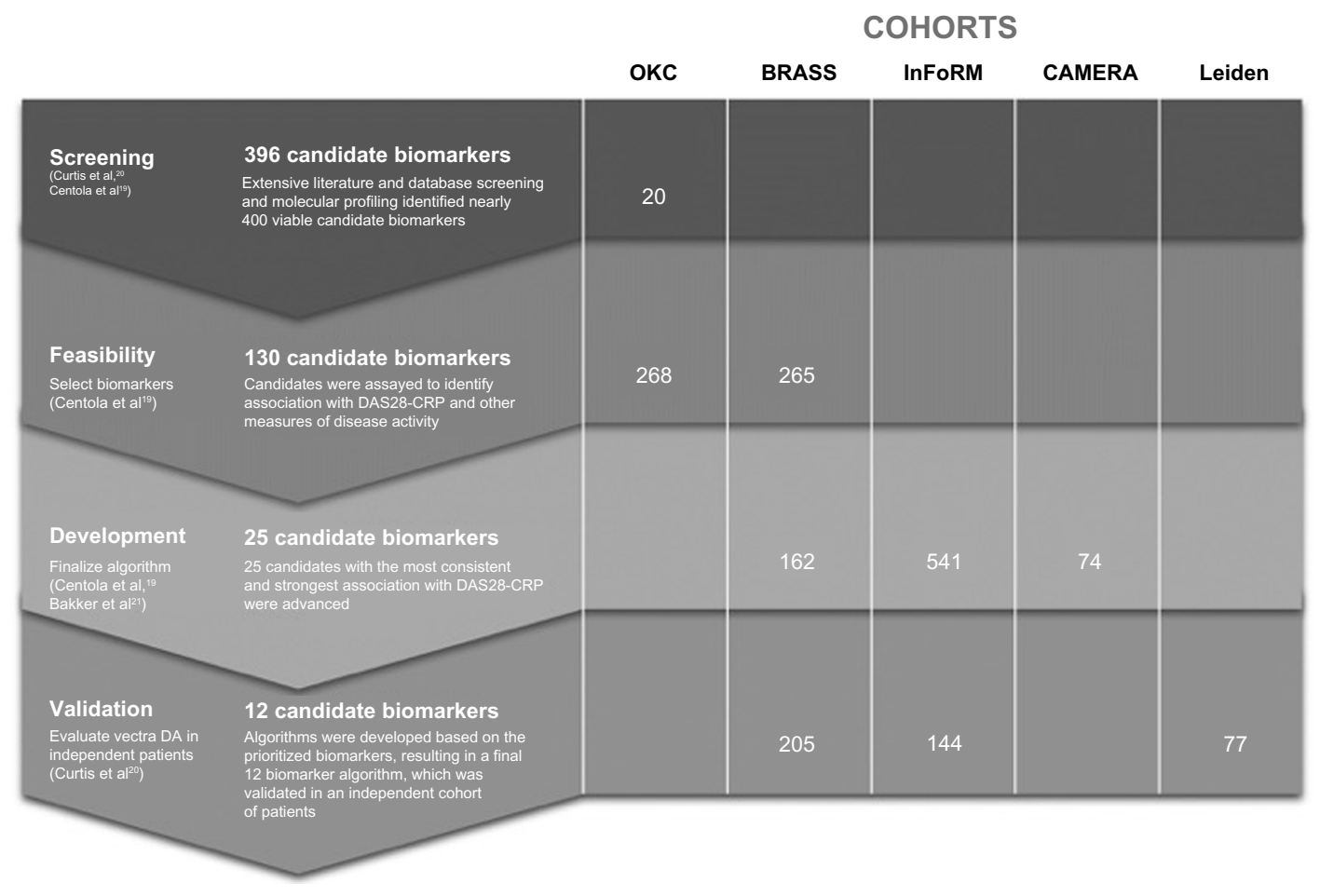

Figure I Description of the multi-step process used in determination of the MBDA score algorithm.

Note: Numbers in column represent number of patients (in some instances, patients might contribute to more than one visit).

Abbreviations: BRASS, Brigham and Women's RA Sequential Study; CAMERA, Computer Assisted Management in Early RA; InFoRM, Index for RA MeasurementCrescendo Bioscience Study; Leiden, Leiden Early Arthritis Cohort; MBDA, multi-biomarker disease activity; OKC, Oklahoma City Community Cohort; DAS28, 28-joint disease activity score; CRP, C-reactive protein; RA, rheumatoid arthritis; DA, disease activity. 
Table I Clinical characteristics of patients based on classification of disease activity by multi-biomarker disease activity score in validation studies

\begin{tabular}{|c|c|c|c|c|c|c|}
\hline & $\mathbf{n}$ & $\begin{array}{l}\text { TJC28 } \\
\text { (mean) }\end{array}$ & $\begin{array}{l}\text { SJC28 } \\
\text { (mean) }\end{array}$ & $\begin{array}{l}\text { PG } \\
\text { (mean) }\end{array}$ & $\begin{array}{l}\text { Median CRP } \\
\text { level (mg/L) }\end{array}$ & $\begin{array}{l}\text { DAS28-CRP } \\
\text { (mean) }\end{array}$ \\
\hline \multicolumn{7}{|l|}{ Seropositive validation } \\
\hline Low: $\leq 29$ & 51 & 3.9 & 3.0 & 29 & 1.4 & 2.65 \\
\hline Moderate: $>29$ to 44 & 66 & 8.3 & 5.9 & 42 & 4.9 & 3.92 \\
\hline High: $>44$ & 113 & 12.0 & 9.1 & 48 & 17.0 & 5.01 \\
\hline \multicolumn{7}{|l|}{ Seronegative validation } \\
\hline Low: $\leq 29$ & 54 & 3.6 & 5.7 & 27 & 0.7 & 2.81 \\
\hline Moderate: $>29$ to 44 & 46 & 5.4 & 7.2 & 41 & 3.9 & 3.73 \\
\hline High: $>44$ & 41 & 9.8 & 11.0 & 52 & 15.0 & 4.98 \\
\hline
\end{tabular}

Note: Reprinted from Curtis JR, van der Helm-van Mil AH, Knevel R, et al. Validation of a novel multi-biomarker test to assess rheumatoid arthritis disease activity. Arthritis Care Res (Hoboken). 2012;64(I2): 1794-1803, copyright @ 2012, with permission from John Wiley and Sons. ${ }^{20}$

Abbreviations: CRP, C-reactive protein; DAS28, 28-joint disease activity score; PG, patient global assessment; SJC28, 28-joint swollen joint count; TJC28, 28-joint tender joint count.

algorithm relative to DAS28-CRP was evaluated by Pearson correlation $(r=0.72 ; P<0.001)$, and area under the receiver operating characteristic curve showed discrimination between patients with moderate/high disease activity and those with remission/low disease activity $(0.86, P<0.001)$. In addition, Bakker et $\mathrm{al}^{21}$ showed that MBDA score, unlike CRP, was an independent predictor of DAS28-CRP as evaluated by multivariate analysis.

\section{Performance of the MBDA score in patients with comorbidities}

The impact of comorbidities on any clinical disease activity assessment tool is important to consider. For example, it is known that acute-phase proteins such as CRP or SAA are a reflection of systemic inflammation. Likewise, cytokines

Table 2 Cross-sectional correlations with additional clinical disease activity measures for the MBDA score and CRPa

\begin{tabular}{|c|c|c|c|c|}
\hline $\begin{array}{l}\text { Biomarker } \\
\text { measure }\end{array}$ & $\begin{array}{l}\text { Clinical } \\
\text { measure }\end{array}$ & $\begin{array}{l}\text { Pearson's } \\
\text { correlation }\end{array}$ & $P$-value & $\mathbf{n}$ \\
\hline \multicolumn{5}{|c|}{ Seropositive validation } \\
\hline MBDA score & SDAI & 0.55 & $<0.001$ & 148 \\
\hline MBDA score & CDAl & 0.48 & $<0.001$ & 148 \\
\hline CRP & CDAI & 0.44 & $<0.001$ & 148 \\
\hline MBDA score & RAPID3 & 0.47 & $<0.001$ & 92 \\
\hline CRP & RAPID3 & 0.37 & $<0.001$ & 92 \\
\hline \multicolumn{5}{|c|}{ Seronegative performance } \\
\hline MBDA score & SDAI & 0.29 & $<0.001$ & 139 \\
\hline MBDA score & CDAl & 0.21 & 0.02 & 139 \\
\hline CRP & CDAI & 0.20 & 0.02 & 139 \\
\hline MBDA score & RAPID3 & 0.26 & 0.003 & 127 \\
\hline CRP & RAPID3 & 0.26 & 0.003 & 127 \\
\hline
\end{tabular}

Note: ${ }^{\text {aCRP }}$ was log-transformed prior to analysis because Pearson's correlation assumes normally distributed data. Reprinted from Curtis JR, van der Helm-van Mil $\mathrm{AH}$, Knevel R, et al. Validation of a novel multi-biomarker test to assess rheumatoid arthritis disease activity. Arthritis Care Res (Hoboken). 2012;64(I2):1794-1803, copyright (C) 2012, with permission from John Wiley and Sons. ${ }^{20}$

Abbreviations: CDAl, clinical disease activity index; CRP, C-reactive protein; MBDA, multi-biomarker disease activity; RAPID3, Routine Assessment of Patient Index Data 3; SDAl, simple disease activity index. such as IL-6 can be elevated in conditions other than RA. To date, limited data have been published on the impact of comorbidities on the MBDA score. The impact of hypertension, osteoporotic fracture, degenerative joint disease, diabetes, and asthma were assessed as part of the development of the MBDA algorithm. ${ }^{19}$ Evaluation of the ratios of median MBDA scores in RA patients with and without comorbidities showed that none of these comorbidities was associated with significant and meaningful influences on the score (Table 3). However, further studies are needed to confirm this and to assess the impact of infections, vaccinations, and other conditions that could potentially affect the score.

\section{Monitoring treatment response with the MBDA score}

It is important that performance of a disease activity scoring system be evaluated in the context of available and forthcoming treatments to demonstrate a change $(\Delta)$ in disease activity over time in treated patients who respond to the treatment, and to determine whether interpretation of the changes is consistent across treatments. A scoring system with objective interpretation guidelines will be valuable for clinicians. Because $\triangle \mathrm{MBDA}$ score reflects the underlying biological changes in treated patients rather than just changes in the signs and symptoms, it is relevant not only to study the score in treated patients, but also to assess its relationships with current measures of treatment response.

The overall association between MBDA score and clinical response has been reported for cohorts of patients treated with different therapeutic modalities, including non-biologic and biologic DMARDs, administered to treatment-naïve patients as well as inadequate responders. The MBDA score has been shown to track disease activity following treatment 
A

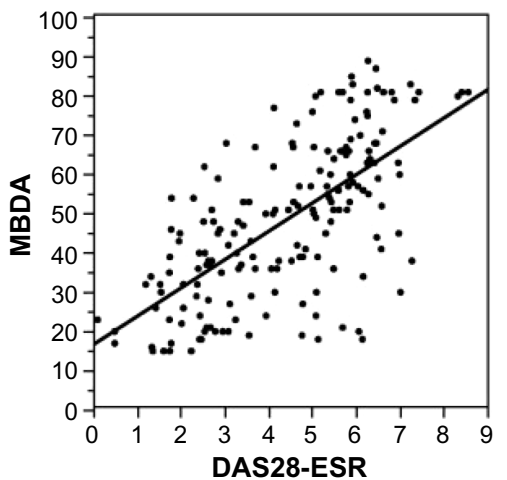

B

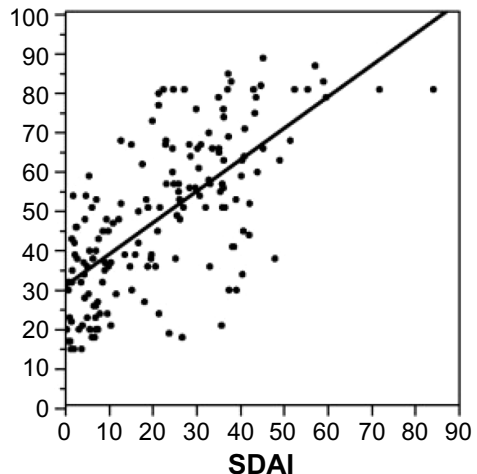

C

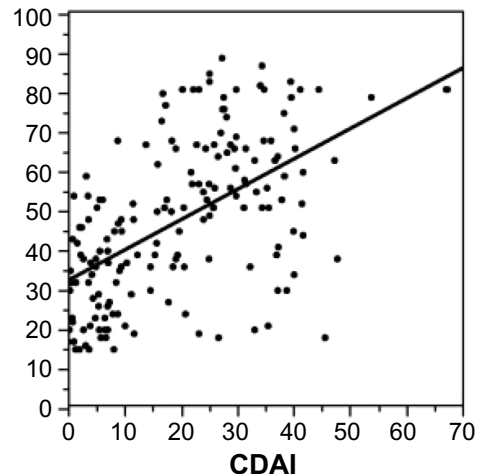

Figure 2 Relationship between the MBDA score and clinical disease activity indices. Correlation and linear regression of MBDA score with DAS28-ESR (A), SDAI (B), and CDAI (C).

Note: Hirata S, Dirven L, Shen Y, et al. A multi-biomarker score measures rheumatoid arthritis disease activity in the BeSt study. Rheumatology. 20I3(7);52:I202-1207, by permission of Oxford University Press. ${ }^{24}$

Abbreviations: CDAl, clinical disease activity index; DAS28, 28-joint disease activity score; ESR, erythrocyte sedimentation rate; MBDA, multi-biomarker disease activity; SDAI, simple disease activity index.

initiation, to distinguish changes in disease activity for patients stratified by EULAR response criteria, and to reflect dose effects in patients treated with a novel therapeutic under investigation..$^{20,21,24,26,27}$ Curtis et al ${ }^{20}$ reported on the $\triangle \mathrm{MBDA}$ score from baseline to the final visit (week 6 or 12) in a group of 45 patients with active disease from the Nested-1 substudy of the Brigham Rheumatoid Arthritis Sequential Study (BRASS), ${ }^{28}$ a prospective observational study of MTX and anti-TNF treatment designed to identify treatment response. In this work, Curtis et al showed that the $\triangle \mathrm{MBDA}$ score was significantly associated with $\triangle \mathrm{DAS} 28$-CRP (Spearman's $r=0.51, P<0.001$ ) and ACR-N (index of improvement of rheumatoid arthritis) scores at week 6 or $12(r=0.45$, $P=0.002) .{ }^{20}$ Furthermore, they showed that $\triangle$ MBDA score

Table 3 Ratios of median disease activity measures ${ }^{\mathrm{a}}$ between RA patients with and without common comorbidities

\begin{tabular}{|c|c|c|c|c|c|}
\hline Comorbidity & n (\%) & CRP & CDAI & $\begin{array}{l}\text { DAS28- } \\
\text { CRP }\end{array}$ & $\begin{array}{l}\text { MBDA } \\
\text { score }\end{array}$ \\
\hline Hypertension & $223(44)$ & 0.98 & $1.32^{\mathrm{b}}$ & $1.14^{\mathrm{b}}$ & 1.05 \\
\hline Osteoarthritis ${ }^{c}$ & $172(34)$ & 0.88 & 1.17 & 1.13 & 1.05 \\
\hline $\begin{array}{l}\text { Osteoporotic bone } \\
\text { fractures }\end{array}$ & $|3|(26)$ & 0.91 & 1.05 & 1.02 & 1.05 \\
\hline $\begin{array}{l}\text { Degenerative joint } \\
\text { disease }^{c}\end{array}$ & $113(22)$ & 1.20 & 1.18 & $1.11^{\mathrm{b}}$ & 1.07 \\
\hline Diabetes & $73(14)$ & I.0I & 1.09 & 1.04 & $1.07^{\mathrm{b}}$ \\
\hline Asthma & $50(10)$ & 1.28 & I.II & 1.05 & 1.05 \\
\hline
\end{tabular}

Notes: ${ }^{a}$ Values close to 1.0 indicate that the measurement or test is not affected by the comorbidity; ${ }^{b}$ nominal $P<0.05$ adjusted for age and sex; when adjusted for multiple comparisons, none was statistically significant; 'osteoarthritis and degenerative joint disease were listed as separate conditions on the case report forms. Reprinted from Centola M, Cavet G, Shen Y, et al. Development of a multi-biomarker disease activity test for rheumatoid arthritis. PLoS One. 20I3;8(4):e60635. ${ }^{19}$

Abbreviations: CDAl, clinical disease activity index; CRP, C-reactive protein; DAS28, 28-joint disease activity score; MBDA, multi-biomarker disease activity; RA, rheumatoid arthritis. could discriminate clinical responders from non-responders. Similarly, in Hirata et al, ${ }^{24}$ a significant correlation between $\triangle$ MBDA score from baseline to 1 year and $\triangle \mathrm{DAS} 28$-ESR was reported for a cohort of 54 patients receiving either MTX or MTX plus infliximab $(r=0.55, P<0.0001)$. A significant correlation was also reported with $\triangle \mathrm{SDAI}(r=0.35, P=0.0158)$ but not with $\triangle$ CDAI $(r=0.18, P>0.05)$. In addition, the authors showed that MBDA scores were associated with ACR/EULAR Boolean remission (28-joint tender joint count (TJC28) $<1,28$-joint swollen joint count (SJC28) $<1$, VAS$\mathrm{GH}$ (visual analog scale-global health) $<1, \mathrm{CRP}<1 \mathrm{mg} / \mathrm{dL}$; $P<0.0001)$ and that remission by MBDA score $(<26)$ was also associated with remission by DAS28-ESR, SDAI, and CDAI. ${ }^{24}$

The ability of the MBDA score to detect changes in response to treatment was also reported in Bakker et al. ${ }^{21}$ In this controlled study, patients with early RA were initially treated with MTX while randomly assigned to either an intensive or a conventional treatment strategy. Analysis of a group of 46 patients with MBDA score at baseline and 6 months showed that the mean \pm standard deviation MBDA score dropped significantly from $53 \pm 18$ to $39 \pm 16$ $(P<0.0001)$. In addition, separate analyses of the intensive and conventional treatment groups showed that there was a significant decrease in MBDA score in the intensive strategy group $(P<0.0001$; Figure 3$)$.

More recently, changes in MBDA score in 147 MTX inadequate response patients treated with TNF inhibitors for a period of 1 year in clinical practice were reported. ${ }^{27}$ A significant association was observed between $\triangle \mathrm{MBDA}$ score and EULAR response, with a greater median decrease in MBDA score reported in EULAR good responders than in 


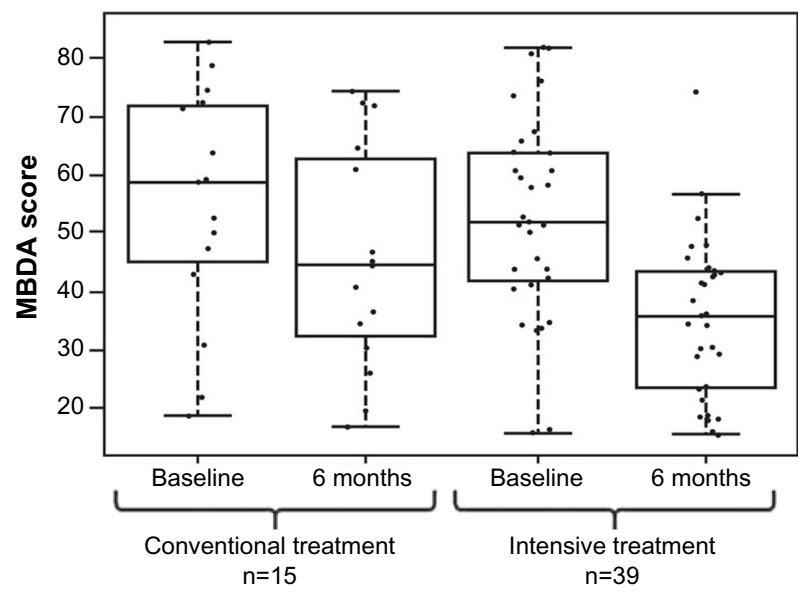

Figure 3 MBDA scores by treatment arm and time point.

Notes: For each treatment strategy (ie, intensive tight control MTX-based [ $n=39]$ and conventional MTX-based [ $n=15]$ treatment strategy), the results of the MBDA score are shown at baseline and after 6 months of treatment. Only results of patients with MBDA score at baseline and 6 months are shown. Reproduced from Bakker MF, Cavet G, Jacobs JW, et al. Performance of a multi-biomarker score measuring rheumatoid arthritis disease activity in the CAMERA tight control study. Ann Rheum Dis. 20 I 2; I ( I0): I692-1697, copyright (C) 20 I2, with permission from BMJ Publishing Group Ltd. ${ }^{2}$

Abbreviations: MBDA, multi-biomarker disease activity; MTX, methotrexate.

EULAR non-responders (Figure 4). A significant decrease in MBDA score and a correlation between $\triangle \mathrm{MBDA}$ score and $\Delta$ DAS28-ESR $(r=0.48)$ and DAS28-CRP $(r=0.46)$ were also reported. In addition, the authors studied the relationships between $\triangle \mathrm{MBDA}$ score and $\triangle \mathrm{DAS} 28-\mathrm{ESR}$ or $\triangle \mathrm{DAS} 28-$ CRP in response to adalimumab, etanercept or infliximab, which were used in the study, and showed that there were no significant differences in the linear relationships between these changes. These findings support the conclusion that the biomarker-based score behaved in a similar manner in patients treated with any of these three TNF inhibitors.

\section{Predicting radiographic damage with the MBDA score}

One of the primary goals of RA treatment has been to prevent or limit joint damage and functional disability. Availability of measurements that would easily identify patients at most risk of radiographic progression and those with lesser risks would allow for a more personalized therapeutic approach, including rapid treatment adjustments, and would contribute to improving patient outcomes. Several publications on the MBDA score have indicated that remission or low disease activity as categorized by the MBDA score was associated with limited radiographic progression in patients with early RA or established RA treated with DMARDs and, conversely, that higher MBDA scores were associated with an increased risk of radiographic progression..$^{25,29,30}$ van der

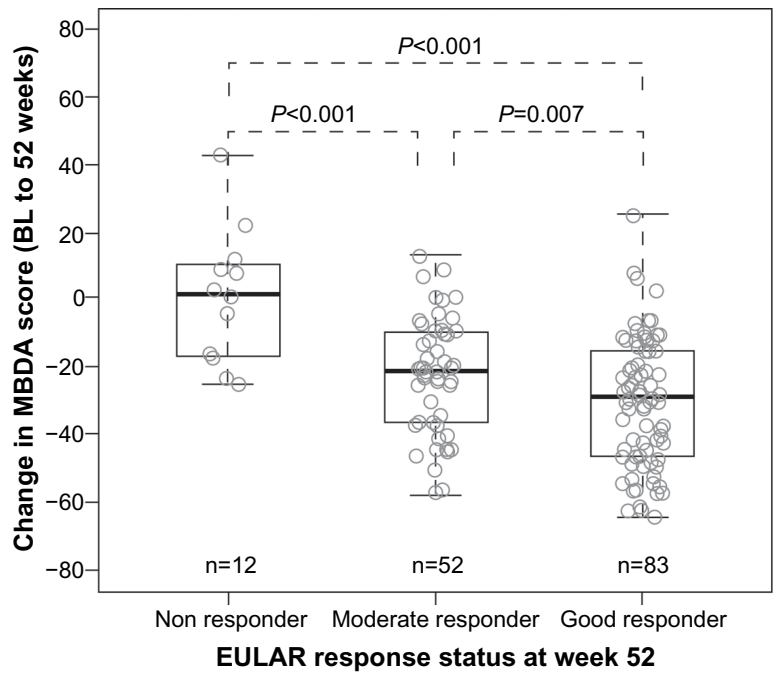

Figure 4 Changes $(\Delta)$ in MBDA score by EULAR response at week 52 . Notes: Box and whisker plot of $\triangle M B D A$ score from baseline to 52 weeks after initiation of TNF inhibitor treatment in patients with no, moderate, and good treatment response at 52 weeks as defined by EULAR criteria. Statistical significance of the difference between the responder groups was assessed by Wilcoxon's ranksum test. Thick horizontal line indicates the median; box indicates the interquartile range (IQR); and whiskers indicate the most extreme points within 1.5 times the IQR from the limits of the box. Hirata S, Li W, Defranoux N, et al.A multi-biomarker disease activity score tracks clinical response consistently in patients with rheumatoid arthritis treated with different anti-tumor necrosis factor therapies: A retrospective observational study. Mod Rheumatol. 2015;25(3):344-349, copyright (C) 20I4, Informa Healthcare. ${ }^{27}$ Reproduced with permission of Informa Healthcare.

Abbreviations: BL, baseline; EULAR, European League Against Rheumatism; IQR, interquartile range; MBDA, multi-biomarker disease activity; TNF, tumor necrosis factor.

Helm-van Mil et al evaluated the radiographic progression of 163 patients (271 visits) in the Leiden Early Arthritis cohort and assessed whether the MBDA score was predictive for risk of radiographic progression. The authors reported that among patients in DAS28-CRP remission $(<2.32)$, having a high ( $>44)$ MBDA score was a significant indicator of elevated risk of radiographic progression over the following 12 months, and the patients were 2.28 (95\% confidence interval $1.13,3.68)$ times as likely to experience joint damage progression. This finding demonstrates the potential clinical utility of a biomarker-based score for detecting patients with subclinical disease activity versus those who are in clinical remission and do not experience radiographic progression. ${ }^{25}$

In a study conducted by Markusse et al, 180 serum samples were analyzed from patients enrolled in the BeSt study. The authors reported that in patients initiating treatment at baseline, the MBDA score predicted radiographic damage progression from baseline to year 1 and from year 1 to year $2 .^{29}$ Similar findings were published by Hambardzumyan et al, who demonstrated that among patients $(n=235)$ from the Swedish Farmacotherapy (SWEFOT) trial, a randomized study of treatment strategies in MTX-naïve 
patients with early RA, the baseline MBDA score was an independent predictor of the risk of rapid radiographic progression (change in Sharp-van der Heijde score $>5$ ) at 1 year, with rapid progression observed in none of the patients with low $(<30)$ MBDA scores, 3\% of those with moderate (30-44) MBDA scores, and $21 \%$ of patients with high (>44) MBDA scores. ${ }^{30}$ The authors also reported that the baseline MBDA score was more effective than baseline CRP or DAS28-ESR at differentiating between rapid progressors and non-progressors (Figure 5).

\section{Reported impact on clinical decision-making}

In addition to reports showing the benefit of the MBDA score as a tool to assess disease activity, a small number of studies have evaluated the impact of the MBDA score on the physician decision-making process. ${ }^{31,32}$ In particular, $\mathrm{Li}$ et al reported that incorporating the MBDA score as part of patient assessment can result in changed treatment plans and treatment decisions by office-based rheumatologists. ${ }^{32}$
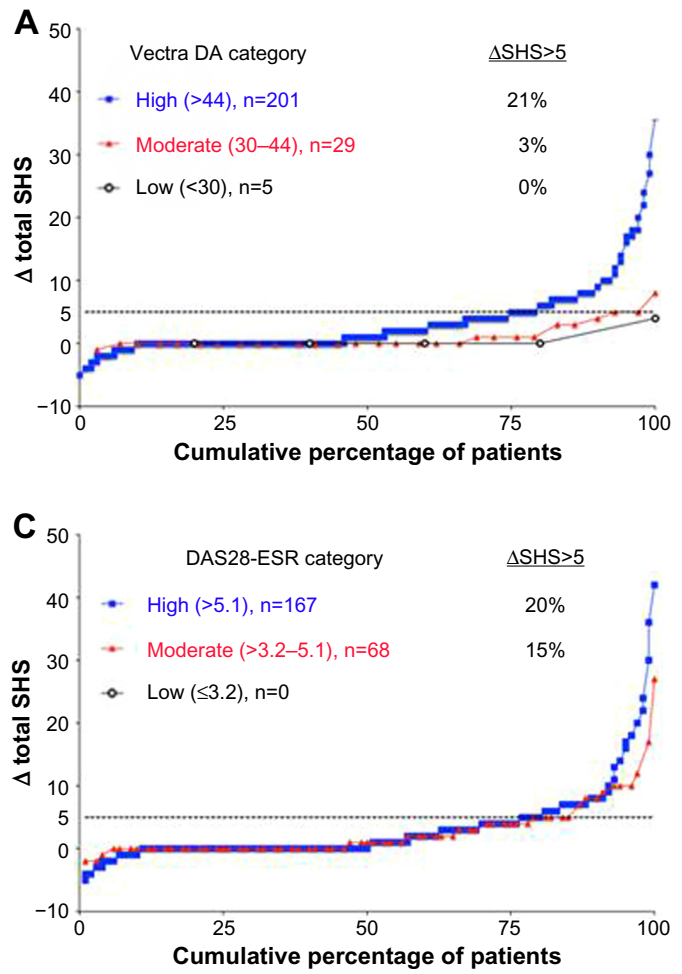

\section{Discussion}

Since the MBDA score is entirely based on concentration of serum markers, one can expect a certain level of discordance at times between this score and clinical disease activity measures. Observed clinical manifestations could be the result of processes other than RA, such as in patients with fibromyalgia or other comorbidities. Preliminary results, published in a review by Segurado and Sasso, indicated that the MBDA score might not be influenced by fibromyalgia and thus may provide a more accurate evaluation of RA disease activity than other composite clinical measures in patients with RA and fibromyalgia. ${ }^{33}$ Use of the MBDA score would thus allow for better management of these patients. In contrast, other conditions that affect the level of inflammatory mediators might also impact the disease activity score; and it would be valuable to see some work exploring the potential impact of cancer, infection, or vaccination on this multi-biomarker-based score. Likewise, it is known that some blood tests, such as ESR, can be influenced by age, sex, or conditions such as anemia, which raises the question of
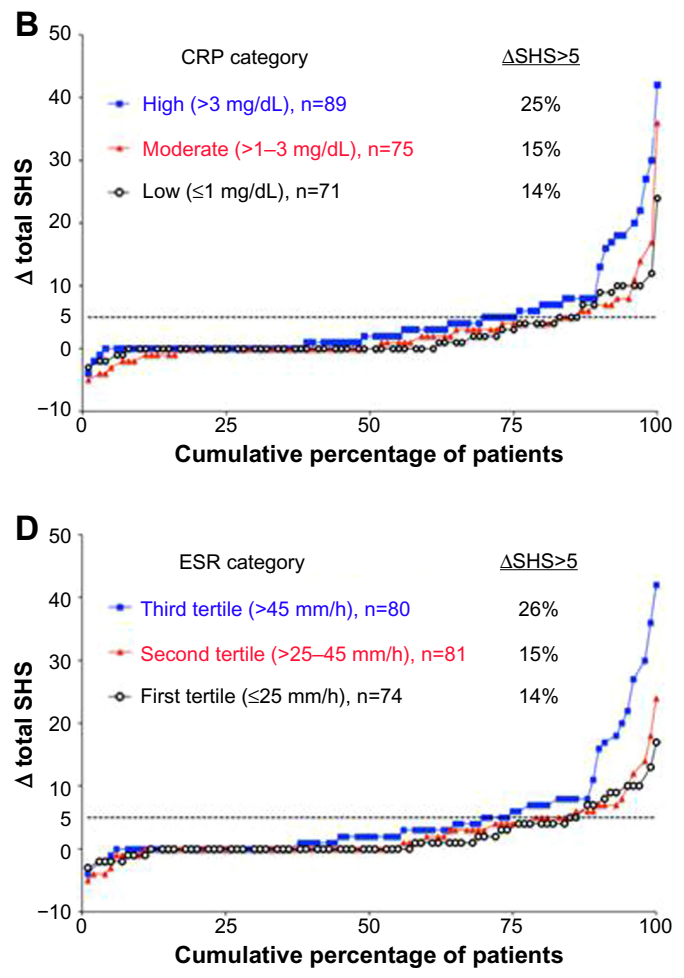

Figure 5 Probability plots of radiographic progression at year I for high, moderate, and low disease activity patient ( $\mathrm{n}=235)$ grouped according to baseline MBDA (A), CRP (B), DAS28 (C), and ESR (D).

Notes: Each black circle represents a patient with low disease activity, the red triangle indicates moderate disease activity, and the blue square indicates high disease activity. Horizontal dashed line represents $\triangle \mathrm{SHS}=5$ from baseline to I year, above which the change is considered as rapid radiographic progression ( $\Delta \mathrm{SHS}>5$ ). Reproduced from Hambardzumyan K, Bolce R, Saevarsdottir S, et al. Pretreatment multi-biomarker disease activity score and radiographic progression in early RA: results from the SWEFOT trial. Ann Rheum Dis. 2015;74(6): I 102-I 109, copyright (C) 2014, with permission from BMJ Publishing Group Ltd. ${ }^{30}$

Abbreviations: CRP, C-reactive protein; DA, disease activity; DAS28, 28-joint disease activity score; ESR, erythrocyte sedimentation rate; MBDA, multi-biomarker disease activity; SHS, Sharp-van der Heijde score. 
whether factors other than comorbidities potentially influence the MBDA score. Although Curtis et al showed that the association between the MBDA score and the DAS28-CRP in subgroups of seronegative or seropositive patients defined by sex, age ( $<65$ versus $\geq 65$ years), body mass index ( $\leq 25$ versus $\left.>25 \mathrm{~kg} / \mathrm{m}^{2}\right)$, or RA therapy, were for the most part statistically significant $(P<0.05),{ }^{20}$ no study to date has been published that reports the influence of sex, age, obesity, or presence of extra-articular manifestations on the MBDA score. Such studies would be useful to provide further context for interpretation of the MBDA score. In view of these considerations, the MBDA score and clinically-based measures provide complementary information for assessing disease activity in patients with RA.

It is also important for a disease activity measure to be consistent in patients regardless of their duration of disease and/or the nature of their treatments. A score based on biomarkers might be particularly influenced by biologic treatment. Calculation of the MBDA score is based on the serum concentration of multiple biomarkers representing different pathways involved in the pathophysiology of RA, a factor that could confer more robustness to the score. It is, however, important to assess the performance of such a disease activity measure under new treatment conditions. Literature on the MBDA score has shown that it tracked changes in disease activity after response to MTX and can therefore be used to assess changes in disease activity. In patients with incomplete response to oral DMARDs, the MBDA score was shown to be consistent in measuring response to different TNF inhibitors. In another study, the MBDA score was found to decrease significantly more $(P=0.008)$ and in a dose-dependent manner $(P<0.001)$ in subjects treated with the investigational drug mavrilimumab than in the placebo group. ${ }^{26}$ Understanding the change in individual biomarkers as well as in the overall score under different conditions would need further confirmation.

A new application for the biomarker-based disease activity measure is in the arena of clinical trial design, where this measure could potentially add value as an inclusion criterion for patient eligibility. The recent medical literature suggests that, contrary to conventional thought, among RA patients who are DMARD-experienced, acutephase reactants such as CRP frequently do not correlate with disease activity measures, including joint counts and global assessments. ${ }^{16}$ Based on this finding, Kay et al speculated that using elevated CRP as an inclusion criterion for clinical trials of experimental RA treatments may exclude some patients with active disease. ${ }^{16}$ Using a high MBDA score as a complementary inclusion criterion might help to identify those patients who would have been otherwise excluded. This approach may lend new insights into RA clinical trial design.

In a 2013 review, Murray and Lopez addressed the challenge of measuring the burden of global disease and the importance of disease-specific metrics. ${ }^{34}$ Such measures have become standard in medical fields from cardiology and diabetes to management of human immunodeficiency virus. In addition to other benefits, objective measures of disease have helped control undertreatment and overtreatment of patients. Rheumatology is lagging in that regard, with limited objective metrics available to practitioners to assess disease activity and treatment response. The development and validation of the MBDA score is a step forward for the field of rheumatology in that it represents an objective tool that can help physicians perform regular objective disease assessments in the clinical setting aimed at the treat-to-target strategy and ultimately resulting in improved patient outcomes.

\section{Acknowledgments}

The authors thank all investigators, the medical staff of all participating institutions, and the laboratory team at Crescendo Bioscience Inc. who have provided helpful findings from MBDA in RA. They also thank Sara Arzeno, who provided medical writing assistance, Rebecca Bolce for critical review and editing, and Arbor Communications Inc. for editorial assistance.

\section{Disclosure}

SH has received consulting fees, speaking fees, and/or honoraria from AbbVie Japan, Eisai, and Bristol-Myers; ND is an employee of Crescendo Bioscience Inc.; KH has no disclosures; KY has received consulting fees and/or honoraria from Pfizer Japan Inc.; YT has received consulting fees, speaking fees, and/or honoraria from Crescendo Bioscience, Mitsubishi-Tanabe, Eisai, Chugai, Abbott Japan, Astellas, Daiichi-Sankyo, AbbVie, Janssen, Pfizer, Takeda, Astra-Zeneca, Eli Lilly Japan, GlaxoSmithKline, Quintiles, MSD, and Asahi-Kasei, and has received research grants from Bristol-Myers, Mitsubishi-Tanabe, AbbVie, MSD, Chugai, Astellas, and Daiichi-Sankyo.

\section{References}

1. Cross M, Smith E, Hoy D, et al. The global burden of rheumatoid arthritis: estimates from the global burden of disease 2010 study. Ann Rheum Dis. 2014;73(7):1316-1322. 
2. Yamanaka H, Sugiyama N, Inoue E, Taniguchi A, Momohara S. Estimates of the prevalence of and current treatment practices for rheumatoid arthritis in Japan using reimbursement data from health insurance societies and the IORRA cohort (I). Mod Rheumatol. 2014;24(1):33-40.

3. Centers for Disease Control and Prevention. Rheumatoid arthritis. Available from: http://www.cdc.gov/arthritis/basics.rheumatoid.htm. Accessed February 9, 2015.

4. Fransen J, Moens HB, Speyer I, van Riel PL. Effectiveness of systematic monitoring of rheumatoid arthritis disease activity in daily practice: a multicentre, cluster randomised controlled trial. Ann Rheum Dis. 2005;64(9):1294-1298.

5. Svensson B, Schaufelberger C, Teleman A, Theander J. Remission and response to early treatment of RA assessed by the Disease Activity Score. BARFOT study group. Better Anti-rheumatic Farmacotherapy. Rheumatology (Oxford). 2000;39(9):1031-1036.

6. Smolen JS, Aletaha D, Bijlsma JW, et al; T2T Expert Committee. Treating rheumatoid arthritis to target: recommendations of an international task force. Ann Rheum Dis. 2010;69(4):631-637.

7. Grigor C, Capell H, Stirling A, et al. Effect of a treatment strategy of tight control for rheumatoid arthritis (the TICORA study): a single-blind randomised controlled trial. Lancet. 2004;364(9430):263-269.

8. Verstappen SM, Jacobs JW, van der Veen MJ, et al. Intensive treatment with methotrexate in early rheumatoid arthritis: aiming for remission. Computer Assisted Management in Early Rheumatoid arthritis (CAMERA, an open-label strategy trial). Ann Rheum Dis. 2007;66(11): 1443-1449.

9. Goekoop-Ruiterman YP, de Vries-Bouwstra JK, Allaart CF, et al. Clinical and radiographic outcomes of four different treatment strategies in patients with early rheumatoid arthritis (the BeSt study): A randomized, controlled trial. Arthritis Rheum. 2005;58(Suppl 2):S126-S135.

10. Anderson J, Caplan L, Yazdany J, et al. Rheumatoid arthritis disease activity measures: American College of Rheumatology recommendations for use in clinical practice. Arthritis Care Res (Hoboken). 2012; 64(5):640-647.

11. Singh JA, Furst DE, Bhart A, et al. 2012 update of the 2008 American College of Rheumatology recommendations for the use of diseasemodifying antirheumatic drugs and biologic agents in the treatment of rheumatoid arthritis. Arthritis Care Res (Hoboken). 2012;64(5): 625-639.

12. Smolen JS, Landewé R, Breedveld FC, et al. EULAR recommendations for the management of rheumatoid arthritis with synthetic and biological disease-modifying antirheumatic drugs: 2013 update. Ann Rheum Dis. 2014;73(3):492-509.

13. Felson DT, Smolen JS, Wells G, et al; European League Against Rheumatism. American College of Rheumatology/European League Against Rheumatism provisional definition of remission in rheumatoid arthritis for clinical trials. Arthritis Rheum. 2011;63(3):573-586.

14. Gibson DS, Finnegan S, Pennington S, et al. Validation of protein biomarkers to advance the management of autoimmune disorders. In: Huang FP, editor. Current Concepts and Advances from Bedside to Mechanistic Insights. Rijeka, Croatia/Shanghai, China: In Tech; 2011. Available at: http://cdn.intechweb.org/pdfs/20666.pdf. Accessed February 9, 2015.

15. Liao H, Wu J, Kuhn E, et al. Use of mass spectrometry to identify protein biomarkers of disease severity in the synovial fluid and serum of patients with rheumatoid arthritis. Arthritis Rheum. 2004;50(12):3792-3803.

16. Kay J, Morgacheva O, Messing SP, et al. Clinical disease activity and acute phase reactant levels are discordant among patients with active rheumatoid arthritis: acute phase reactant levels contribute separately to predicting outcome at one year. Arthritis Res Ther. 2014;16:R40.
17. Willemze A, Toes RE, Huizinga TW, et al. New biomarkers in rheumatoid arthritis. Neth J Med. 2012;70(9):392-399.

18. Chandrashekara S. Current studies of biomarkers for the early diagnosis of rheumatoid arthritis. Curr Biomark Find. 2014;4:107-119.

19. Centola M, Cavet G, Shen Y, et al. Development of a multi-biomarker disease activity test for rheumatoid arthritis. PLoS One. 2013;8(4): e60635.

20. Curtis JR, van der Helm-van Mil AH, Knevel R, et al. validation of a novel multibiomarker test to assess rheumatoid arthritis disease activity. Arthritis Care Res (Hoboken). 2012;64(12):1794-1803.

21. Bakker MF, Cavet G, Jacobs JW, et al. Performance of a multi-biomarker score measuring rheumatoid arthritis disease activity in the CAMERA tight control study. Ann Rheum Dis. 2012;71(10):1692-1697.

22. Eastman PS, Manning WC, Qureshi F, et al. Characterization of a multiplex, 12-biomarker test for rheumatoid arthritis. JPharm Biomed Anal. 2012;70:415-424.

23. Inoue E, Yamanaka H, Hara M, Tomatsu T, Kamatani N. Comparison of Disease Activity Score (DAS)28-erythrocyte sedimentation rate and DAS28-C-reactive protein threshold values. Ann Rheum Dis. 2007;66(3):407-409.

24. Hirata S, Dirven L, Shen Y, et al. A multi-biomarker score measures rheumatoid arthritis disease activity in the BeSt study. Rheumatology (Oxford). 2013(7);52:1202-1207.

25. Van der Helm-van Mil AH, Knevel R, Cavet G, Huizinga TW, Haney DJ. An evaluation of molecular and clinical remission in rheumatoid arthritis by assessing radiographic progression. Rheumatology (Oxford). 2013;52(5):839-846.

26. Burmester G, Weinblatt M, McInnes I, et al; EARTH Study Group. Efficacy and safety of mavrilimumab in subjects with rheumatoid arthritis. Ann Rheum Dis. 2013;72(9):1445-1452.

27. Hirata S, Li W, Defranoux N, et al. A multi-biomarker disease activity score tracks clinical response consistently in patients with rheumatoid arthritis treated with different anti-tumor necrosis factor therapies: A retrospective observational study. Mod Rheumatol. 2015;25(3): 344-349.

28. Iannaccone CK, Lee YC, Cui J, et al. Using genetic and clinical data to understand response to disease-modifying anti-rheumatic drug therapy: data from the Brigham and Women's Hospital Rheumatoid Arthritis Sequential Study. Rheumatology (Oxford). 2011;50(1):40-46.

29. Markusse IM, Dirven L, van den Broek M, et al. A multibiomarker disease activity score for rheumatoid arthritis predicts radiographic joint damage in the BeSt study. $J$ Rheumatol. 2014;41(11):2114-2119.

30. Hambardzumyan K, Bolce R, Saevarsdottir S, et al. Pretreatment multi-biomarker disease activity score and radiographic progression in early RA: results from the SWEFOT trial. Ann Rheum Dis. 2015; 74(6):1102-1109.

31. Peabody JW, Strand V, Shimkhada R, Lee R, Chernoff D. Impact of Rheumatoid Arthritis Disease Activity Test on Clinical Practice. PLoS One. 2013;(8)5:e63215.

32. Li W, Sasso EH, Emerling D, Cavet G, Ford K. Impact of a multibiomarker disease activity test on rheumatoid arthritis treatment decisions and therapy use. Curr Med Res Opin. 2013;29(1):85-92.

33. Segurado OG, Sasso EH. Vectra DA for the objective measurement of disease activity in patients with rheumatoid arthritis. Clin Exp Rheumatol. 2014;32 Suppl 85(5):S29-S34.

34. Murray CJ, Lopez AD. Measuring the global burden of disease. N Engl J Med. 2013;369(5):448-457. 


\section{Supplementry material}

Table SI Twelve biomarkers reflect the heterogeneity of RA

\begin{tabular}{lll}
\hline Biomarker & Biomarker category & Primary role \\
\hline VCAM-I & Adhesion molecules & $\begin{array}{l}\text { Cellular influx and tissue } \\
\text { expansion } \\
\text { Cellular influx and tissue } \\
\text { expansion }\end{array}$ \\
IL-6, TNF-RI & $\begin{array}{l}\text { Cytokine-related } \\
\text { proteins }\end{array}$ & $\begin{array}{l}\text { and destruction } \\
\text { Cartilage degradation } \\
\text { and joint damage }\end{array}$ \\
MMP-I, MMP-3 & $\begin{array}{l}\text { Matrix } \\
\text { metalloproteinases }\end{array}$ & $\begin{array}{l}\text { Stromal activity and } \\
\text { regulation (fibroblasts, } \\
\text { chondrocytes, } \\
\text { vascular cells) }\end{array}$ \\
& $\begin{array}{l}\text { Skeletal-related } \\
\text { proteins }\end{array}$ & $\begin{array}{l}\text { Systemic inflammatory } \\
\text { response }\end{array}$ \\
& Hormones & $\begin{array}{l}\text { Systemic inflammatory } \\
\text { response }\end{array}$ \\
\hline
\end{tabular}

Abbreviations: CRP, C-reactive protein; EGF, epidermal growth factor; IL-6, interleukin 6; MMP, matrix metalloproteinase; SAA, serum amyloid A; TNF$R I$, tumor necrosis factor alpha receptor IA; VCAM-I, vascular cell adhesion molecule I; VEGF-A, vascular endothelial growth factor A; YKL-40, human cartilage glycoprotein 39; RA, rheumatoid arthritis.

\section{Publish your work in this journal}

Current Biomarker Findings is an international, peer-reviewed, open access journal publishing original research, reports, reviews and commentaries on all areas of biomarker research. The manuscript management system is completely online and includes a very quick and fair

peer-review system. Visit http://www.dovepress.com/testimonials.php to read real quotes from published authors. 